Pacific Journal of Mathematics

GLEASON'S THEOREM FOR TYPE I YON NEUMANN 


\title{
GLEASON'S THEOREM FOR TYPE I VON NEUMANN ALGEBRAS
}

\author{
JÜRGEN TISCHER
}

For a von Neumann algebra $\mathfrak{A}$ a $G$-measure $m$ on $\mathfrak{A}$ is defined as a map from the projections of $\mathfrak{A}$ to the positive reals which satisfies the equation

$$
m\left(\sum P_{i}\right)=\sum m\left(P_{\imath}\right)
$$

for every family $\left(P_{i}\right)$ of pairwise orthogonal projections. We prove the following generalization of Gleason's theorem: If $m$ is a $G$-measure on a type $I$ von Neumann algebra $\mathfrak{A}$ not containing a type $I_{2}$ direct summand, then there exists an extension of $m$ to a positive normal linear for on $\mathfrak{A}$.

o. Let $H$ be a complex Hilbert space, $\mathfrak{A}$ a von Neumann algebra acting on $H$, and $\mathscr{P}(\mathfrak{A})$ the lattice of projections in $\mathfrak{A}$. A map $m$ defined on $\mathscr{P}(\mathfrak{U})$ with values in the positive reals is called a $G$-measure on $\mathfrak{A}$ if for every orthogonal family $\left(P_{i}\right)$ in $\mathscr{P}(\mathfrak{U})$ the equation $m\left(\sum P_{i}\right)=\sum m\left(P_{i}\right)$ is satisfied. If the equation holds for finite orthogonal families then $m$ is called a finitely additive $G$-measure.

Gleason proved [3] that for $\mathfrak{A}=L(H)$ (the bounded operators on $H$ ) and $\operatorname{dim} H \neq 2$ every $G$-measure is extendable to a (necessary unique and normal) linear form on $\mathfrak{A}$. A. A. Lodkin generalized this result to all von Neumann algebras acting on a separable Hilbert space and not having a type $\mathrm{I}_{2}$ direct summand, but the proofs are only sketched and seem to be incomplete. This paper provides a complete proof of Gleason's theorem for those type I von Neumann algebras not having a type $I_{2}$ direct summand, so in particular no assumption about the separability of $H$ is made.

THeORem 1. Let $\mathfrak{A}$ be a type I von Neumann algebra without a type $\mathrm{I}_{2}$ direct summand. Let $m$ be a G-measure on $\mathfrak{A}$. Then there is an'extension of $m$ to a linear form on $\mathfrak{A}$, which is necessarily normal and unique.

The proof of the theorem is given in a series of lemmas. First we introduce some notation. Throughout the paper let $X$ be a hyperstonean space. For every real or complex Hilbert space $K$ of finite dimension $n$ the space $C(X, K)$ of continuous functions from $X$ to $K$ is an $n$-dimensional module over $C(X)$. We can easily carry 
over many concepts known in the Hilbert space $K$ to the module $C(X, K)$ by defining them pointwise, so let an inner product on $C(X, K)$ with values in $C(X)$ be defined by $(f, g)(x)=(f(x), g(x))$. Two elements $f$ and $g$ are said to be orthogonal to each other $(f \perp g)$ iff $(f, g)=0$. A module basis $\left\{f_{1}, \cdots, f_{m}\right\}$ is called an orthonormal basis if $\left(f_{i}, f_{j}\right) \equiv \delta_{i j}$. To avoid confusion, we define the function | . | from $C(X, K)$ to $C(X)$ by $|f|(x)=\|f(x)\|$, leaving $\|$. $\|$ for the usual supremum norm on $C(X, K)$.

On $C(X, K)$ we define an equivalence relation $\sim$ by $f \sim g$ iff there is a $\varphi \in C(X)$ such that $\varphi(x) \neq 0$ for all $x \in X$ and $f=\varphi g$. Note that the following are equivalent: (i) $f \perp g$; (ii) there exists $g^{\prime} \sim g$ and $f \perp g^{\prime}$; (iii) for all $g^{\prime} \sim g$ the equation $f \perp g^{\prime}$ is satisfied.

For the case $K=\boldsymbol{R}^{3}$ define a cross product $\times$ on $C\left(X, \boldsymbol{R}^{3}\right)$ by $f \times g(x)=f(x) \times g(x)$ (ordinary cross product in $\boldsymbol{R}^{3}$ ). By $S^{2}$ we denote the usual unit sphere in $\boldsymbol{R}^{3}$.

Now we are ready to generalize Gleason's notion of a frame function on $S^{2}$ to the case of a function on $C\left(X, S^{2}\right)$ and to prove that every frame function on $C\left(X, S^{2}\right)$ is continuous. Note that for $X$ a singleton this is just the essential lemma of Gleason's proof. We have tried to follow as closely as possible the outline of Varadarajan's proof [5]. The proofs of Lemmas 7 and 10 are almost exactly the same but are included for the convenience of the reader since they are short. On the other hand, the remainder of the proof requires substantially different arguments in several places.

Definition 2. A function $\phi: C\left(X, S^{2}\right) \rightarrow R^{+}$is called a frame function if there is a positive real constant $W$, called the weight of $\phi$, such that for every orthonormal basis $\left\{f_{1}, f_{2}, f_{3}\right\}$ of $C\left(X, \boldsymbol{R}^{3}\right)$ the equation $\phi\left(f_{1}\right)+\phi\left(f_{2}\right)+\phi\left(f_{3}\right)=W$ is satisfied.

So in particular if $f, g \in C\left(X, S^{2}\right)$ and $f \sim g$, then $\phi(f)=\phi(g)$.

Notation 3. Let $f \in C\left(X, S^{2}\right)$. Then we define

$$
E(f)=\left\{g \in C\left(X, S^{2}\right) \mid g \perp f\right\} ;
$$

$L(f)=\left\{g \in C\left(X, S^{2}\right) \mid \forall x \in X \forall g^{\prime} \in C\left(X, S^{2}\right): g^{\prime} \sim g \Longrightarrow f(x) \neq g^{\prime}(x)\right\} ;$

$$
N(f)=L(f) \cap \cap\{L(h) \mid h \in E(f)\} .
$$

For $f$ and $g$ in $C\left(X, S^{2}\right)$ we have $E(f) \subseteq L(f)$ and $N(f) \subseteq L(f)$. Furthermore, if one notes that $X$ is hyperstonean, the following are easily seen to be equivalent: (i) $f \sim g$; (ii) $E(f)=E(g)$; (iii) $L(f)=$ $L(g)$; (iv) $N(f)=N(g)$. Now let $h$ be in $L(f)$. Then $|h \times f|$ is 
strictly positive on $X$, so $1 /|h \times f|$ is in $C(X)$. Define $h \circ f=1 / \mid h \times$ $f \mid(h \times f)$; then $h \circ f$ is in both $C\left(X, S^{2}\right)$ and $E(f) \cap E(h)$. In particular $h \in L(h \circ f)$, so $h \circ(h \circ f)$ is defined. We write

$$
E W(h, f)=E(h \circ(h \circ f)) \text {. }
$$

REMARK. The notations $E(f), N(f)$ and $E W(h, f)$ are intended to bring to mind the notions equator with respect to $f$, northern hemisphere with respect to $f$ and east-west great circle through $h$ of Gleason's proof; the exact analogy breaks down if $X$ is a singleton.

I. In this section we prove the continuity of a frame function.

Lemma 4. Let $f \in C\left(X, S^{2}\right)$, and $\phi$ be a frame function on $C\left(X, S^{2}\right)$ which is constant on $E(f)$. Then for any $g$ in $N(f)$ and any $h$ in $E W(g, f)$,

$$
\phi(g) \leqq \phi(h)+\phi(f)
$$

Proof. Denote by $k$ the constant value of $\phi$ on $E(f)$, and let $W$ be the weight of $\phi$.

(a) For $l \in L(f)$, we claim that $\phi(l) \leqq k+\phi(f)$.

To see this, note that $\{l, l \circ f,(l \circ f) \circ l\}$ and $\{f, l \circ f,(l \circ f) \circ f\}$ are both orthonormal bases. Moreover $l \circ f$ and $(l \circ f) \circ f$ are in $E(f)$, so

$$
W=\dot{\phi}(f)+\phi(l \circ f)+\phi((l \circ f) \circ f)=\phi(f)+2 k
$$

and

$$
W=\dot{\phi}(l)+\dot{\phi}(l \circ f)+\dot{\phi}((l \circ f) \circ l) \geqq \dot{\phi}(l)+k .
$$

It follows that $\phi(l) \leqq \phi(f)+k$.

(b) For $g \in N(f)$ and $h \in E W(g, f)=E(g \circ(g \circ f))$ define $h^{\prime}=h \circ$ $(g \circ(g \circ f))$. Then $\{g, g \circ f, g \circ(g \circ f)\}$ and $\left\{h^{\prime}, h, g \circ(g \circ f)\right\}$ are orthonormal bases and $g \circ f \in E(f)$, so

$$
\phi\left(h^{\prime}\right)+\phi(h)=\phi(g)+\phi(g \circ f)=\phi(g)+k .
$$

Moreover $h^{\prime} \in L(f)$. Assume the contrary. Then there is an $x \in X$ such that $h^{\prime}(x)= \pm f(x)$ so $f(x) \perp g \circ(g \circ f)(x)$; that is, $\{f(x), g \circ f(x)$, $g \circ(g \circ f)(x)\}$ and $\{g(x), g \circ f(x), g \circ(g \circ f)(x)\}$ are orthonormal bases in $\boldsymbol{R}^{3}$. So $g(x)= \pm f(x)$ which contradicts the fact that $g$ is in $N(f)$.

Since $h^{\prime} \in L(f)$, part (a) yields the inequality

$$
\begin{aligned}
& \phi\left(h^{\prime}\right) \leqq k+\phi(f), \text { which, combined with (1), gives } \\
& \phi(h) \geqq \phi(g)-\phi(f) . \quad \text { This completes the proof. }
\end{aligned}
$$


For the moment let $X$ be a singleton and identify $C\left(X, S^{2}\right)$ with $S^{2}$, so the meaning for points in $S^{2}$ of the notation introduced in 3 is clear. Using cartesian coordinates in $R^{3}$, define $P$ to be the plane $\left\{x_{3}=1\right\}$ in $\boldsymbol{R}^{3}, N=\left\{x \in S^{2} \mid 0<x_{3}<1\right\}$ and $p=(0,0,1) \in S^{2}$. Denote by $\Pi$ the central projection with center 0 of $N$ into $P$. Then $\Pi(p)=$ $p$ and $I I$ is a homeomorphism of $N$ onto $P \backslash\{p\}$ which maps the circles in $N$ with centers $p$ bijectively onto the circles in $E$ with center $p$. If $K \neq E(p)$ is a great circle in $S^{2}$ not containing $p$ then $\Pi(K \cap N)$ is a straight line in $P$ not containing $p$. The mapping so defined is also a bijection. Moreover for $y$ in $N$ a great circle $K$ is equal to $E W(y, p)$ iff $\Pi(K \cap N)$ is perpendicular to the straight line connecting $p$ and $\Pi(y)$.

For $y \in N$ define $G_{y}=\Pi(E W(y, p) \cap N)$, and for $s \in P \backslash\{p\}$ define $G_{s}=G_{\Pi^{-1}(s)}$. Then for $s \in P \backslash\{p\}, G_{s}$ is the unique line in $P$ containing $s$ such that the map $x \rightarrow\|x-p\|$ attains its infimum in $s$.

LeMma 5. Let $X$ be a singleton, $p$ be defined as above, $\alpha \in] 0, \pi / 2\left[\right.$, and $z=(\cos \alpha, 0, \sin \alpha) \in S^{2}$. Define $K_{\alpha}$ to be the open disc in $P$ with center $1 / 2(\Pi(z)+p)$ and radius $\|1 / 2(\Pi(z)-p)\|$, and define $S_{\alpha}$ to be the boundary of $K_{\alpha}$. Then, when $s \in K_{\alpha}$, the intersection of $G_{s}$ and $S_{\alpha}$ consists of two points $I_{1}(s, \alpha)$ and $I_{2}(s, \alpha)$ where $I_{1}$ and $I_{2}$ are continuous functions of $s$ and $\alpha$. Moreover,

$$
z \in E W\left(\Pi^{-1}\left(I_{i}(s, \alpha)\right), p\right) \text { and } \Pi^{-1}\left(I_{i}(s, \alpha)\right) \in E W\left(\Pi^{-1}(s), p\right) \text {. }
$$

Proof. $\Pi(z)$ is of the form $\Pi(z)=(\rho(\alpha), 0,1)$, where $\rho$ is continuous. If $s$ is in $K_{\alpha}$, then $\left(s_{1}-\rho(\alpha) / 2\right)^{2}+s_{2}^{2}<(\rho(\alpha) / 2)^{2}$, so $s_{1} \rho(\alpha) /$ $\left(s_{1}^{2}+s_{2}^{2}\right)-1>0$. Now

$$
S_{\alpha}=\left\{x \in P \mid x_{1}^{2}+x_{2}^{2}-x_{1} \rho(\alpha)=0\right\}
$$

and

$$
G_{s}=\left\{x \in \boldsymbol{R}^{3} \mid \exists \mu \in \boldsymbol{R} \text { such that } x=\left(s_{1}, s_{2}, 1\right)+\mu\left(-s_{2}, s_{1}, 0\right)\right\} .
$$

Thus for the points $s$ in $G_{s} \cap S_{\alpha}$ we have

$$
\mu^{2}+\mu\left(\frac{s_{2} \rho(\alpha)}{s_{1}^{2}+s_{2}^{2}}\right)-\left(\frac{s_{1} \rho(\alpha)}{s_{1}^{2}+s_{2}^{2}}-1\right)=0
$$

that is, there are exactly two such points. Let $I_{1}(s, \alpha)\left(\right.$ resp. $I_{2}(s, \alpha)$ ) be the point corresponding to the positive (resp. negative) square root of $\mu^{2}$; then $I_{1}$ and $I_{2}$ are continuous functions of $s$ and $\alpha$. Since $I_{i}(s, \alpha)$ is in $G_{s}$, it follows that $\Pi^{-1}\left(I_{i}(s, \alpha)\right)$ is in $E W\left(\Pi^{-1}(s), p\right)$. Let $K$ be the disc in $P$ with center $p$ and radius $\|\Pi(z)-p\|$, and let $G$ be the line containing $\Pi(z)$ and either $I_{1}(s, \alpha)$ or $I_{2}(s, \alpha)$, say $I_{1}(s, \alpha)$. Then $I_{1}(s, \alpha)$ is the midpoint of $G \cap K$, so $G=G_{I_{1}(s, \alpha)}$ and $z \in$ 
$E W\left(\Pi^{-1}\left(I_{1}(s, \alpha), p\right)\right.$.

LEMma 6. Let $f \in C\left(X, S^{2}\right)$ and $\phi$ be a frame function defined on $C\left(X, S^{2}\right)$ and constant on $E(f)$. Let $g \in N(f)$, and define $M_{g}=$ $\{h \in N(f) \mid \exists l \in N(f) \cap E W(h, f)$ such that $g \in E W(l, f)\}$. Then the interior of $M_{g}$ is nonempty and for all $h \in M_{g}$ we have:

$$
\phi(h) \leqq \phi(g)+2 \phi(f) \text {. }
$$

Proof. The inequality is an immediate consequence of Lemma 4 and the definition of the set $M_{g}$, so we only have to show that the interior of $M_{g}$ is nonempty.

(a) First we show that we can assume that $f \equiv(0,0,1)$. With respect to the canonical module basis of $C\left(X, R^{3}\right)$ we can write $f=$ $\left(f_{1}, f_{2}, f_{3}\right)$, where the $f_{i}$ 's are continuous functions on $X$ and $f_{1}^{2}+$ $f_{2}^{2}+f_{3}^{2}=1$. Define $A=\left\{x \in X \mid f_{3}^{2} \neq 1\right\}$, and define $\alpha^{\prime}, \beta^{\prime}: A \rightarrow \boldsymbol{R}$ by $\alpha^{\prime}(x)=\left(1-f_{3}^{2}(x)\right)^{-1 / 2} f_{1}(x)$ and $\beta^{\prime}(x)=\left(1-f_{3}^{2}(x)\right)^{-1 / 2} f_{2}(x)$. Then $\alpha^{\prime}$ and $\beta^{\prime}$ are continuous and bounded and $X$ is hyperstonean, so there are continuous extensions $\alpha$ and $\beta$ on cl $A$, the closure of $A$. Using the canonical basis of $\boldsymbol{R}^{3}$ we define $U^{\prime}: X \rightarrow L\left(\boldsymbol{R}^{3}\right)$ by

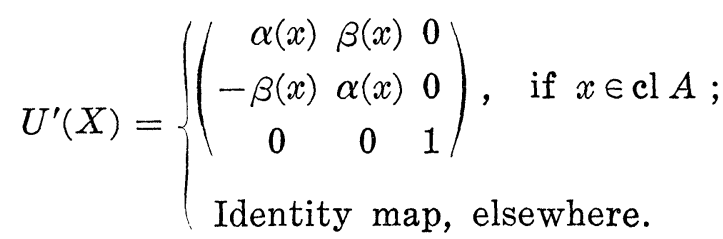

Since cl $A$ is open and closed the components $U_{i j}^{\prime}$ of $U^{\prime}$ are continuous functions on $X$. The mapping $U^{\prime}$ induces an isomorphism $U$ of the vector space $C\left(X, R^{3}\right)$ by $U h(x)=U^{\prime}(x) h(x)$. For all $h$ in $C\left(X, R^{3}\right)$ we have $|U h|=|h|$ and $\|U h\|=\|h\|$; that is, $U$ is an isometry. Moreover, for all $h, l$ in $C\left(X, \boldsymbol{R}^{3}\right)$ we have that $h \perp l$ iff $U h \perp U l$, so $U$ maps $C\left(X, S^{2}\right)$ homeomorphically onto itself, taking $M_{g}$ onto $M_{U g}$. Furthermore $U f$ is of the form $U f=\left((U f)_{1}, 0, f_{3}\right)$. Define $W: X \rightarrow L\left(\boldsymbol{R}^{3}\right)$ by

$$
W(x)=\left(\begin{array}{ccc}
f_{3}(x) & 0 & -(U f)_{1}(x) \\
0 & 1 & 0 \\
(U f)_{1}(x) & 0 & f_{3}(x)
\end{array}\right) .
$$

Then $W$ has the same properties as $U$ and $W(U f) \equiv(0,0,1)$.

(b) Now we show that we can make the additional assumption that $g$ is of the form $g(x)=(\cos \alpha(x), 0, \sin \alpha(x))$, where $\alpha$ is a continuous function on $X$ with values in $] 0, \pi / 2[$. Since $f \equiv(0,0,1), g$ is in $N(f)$ iff $\left.g_{3}(x) \in\right]-1,0[\cup] 0,1[$ for all $x$ in $X$. Reasoning as in 
(a) we can assume that $g$ is of the form $g=\left(g_{1}, 0, g_{3}\right)$. Now the function $x \rightarrow \operatorname{sign}\left(g_{3}(x)\right)$ is a continuous function from $X$ to $\{-1,1\}$ and $g^{\prime}=\operatorname{sign}\left(g_{3}\right) \cdot g$ is equivalent to $g$, so $M_{g^{\prime}}=M_{g}$. Moreover we have $0<g_{3}^{\prime}(x)<1$ for all $x$ in $X$. Finally define $\left.\alpha: X \rightarrow\right] 0, \pi / 2[$ by $\alpha(x)=\arcsin g_{3}^{\prime}(x)$.

(c) Let $\left.\alpha_{0}=\sup \alpha(x) \in\right] 0, \pi / 2\left[\right.$, and define $z_{0} \in S^{2}$ by $z_{0}=\left(\cos \alpha_{0}\right.$, $\left.0, \sin \alpha_{0}\right)$. Let $K$ be the open disc in the plane $P$ with center $1 / 2\left(\Pi\left(z_{0}\right)+p\right)$ and radius $\left\|1 / 2\left(\Pi\left(z_{0}\right)-p\right)\right\|$. Let $B=C\left(X, \Pi^{-1}(K)\right)$; then $B$ is an open subset of $C\left(X, S^{2}\right)$. Choose $h \in B$; with the notation of Lemma 5 , we have $\Pi(h(x)) \in K_{\alpha(x)}$ for every $x$ in $X$. Define $l: X \rightarrow S^{2}$ by $l(x)=\Pi^{-1}\left(I_{1}(\Pi(h(x), \alpha(x)))\right)$. It follows from Lemma 5 that $l \in C\left(X, S^{2}\right), g \in E W(l, f)$, and $l \in E W(h, f)$. Moreover $0<l_{3}(x)<$ 1 and $0<h_{3}(x)<1$ for all $x$ in $X$, so $h$ is in $M_{g}$. We conclude that $U \subseteq M_{g}$, which completes the proof of the lemma.

Lemma 7. With $f$ and $\phi$ defined as in Lemma 6, let $\eta$ be a positive real number with $\phi(f)<\eta$. Then there is a $g$ in $N(f)$ and an open set $M$ containing $g$ such that

$$
0 \leqq \sup \phi(M)-\inf \dot{\phi}(M) \leqq 3 \eta
$$

Proof. (The proof follows exactly the proof of Lemma 7.16, p. 152 of [5].) Define $b=\inf \phi(N(f)) \geqq 0$. Let $l$ be in $N(f)$ such that $b \leqq \phi(l) \leqq b+\eta$. By Lemma 6 we have $\phi(k) \leqq \phi(l)+2 \eta$ for every $k$ in $M_{l}$, so $b \leqq \phi(k) \leqq b+3 \eta$. Define $M$ to be the interior of $M_{l}$ and let $g$ be any point of $M$.

Lemma 8. Let $f$ be in $C\left(X, S^{2}\right), \varepsilon>0$, and

$$
M(f, \varepsilon)=\left\{g \in C\left(X, S^{2}\right) \mid \forall f^{\prime} \sim f \forall x \in X\left\|g(x)-f^{\prime}(x)\right\|>\varepsilon\right\} .
$$

Then there is a constant $k(\varepsilon)$ depending only on $\varepsilon$ such that for all $h_{1}, h_{2}$ in $M(f, \varepsilon)$ we have

$$
\left\|h_{1} \circ f-h_{2} \circ f\right\| \leqq k(\varepsilon)\left\|h_{1}-h_{2}\right\| .
$$

Proof. For $x$ in $X$ and $i=1,2$ define $K(x, i)$ to be the great circle in $S^{2}$ containing $f(x)$ and $h_{i}(x)$. Define $M(x, \varepsilon)=\left\{t \in S^{2} \mid\|f(x)-t\|\right.$ $>\varepsilon$ and $\|-f(x)-t\|>\varepsilon\}$. Let $A(x, i)$ be that component of $K(x, i) \cap$ $M(x, \varepsilon)$ which contains $h_{i}(x)$, and let $v_{i}(x)$ be the point of intersection of $A(x, i)$ and $E(f(x))$. Then

$$
\begin{aligned}
\left\|h_{1}(x) \circ f(x)-h_{2}(x) \circ f(x)\right\| & =\left\|v_{1}(x) \times f(x)-v_{2}(x) \times f(x)\right\| \\
& =\left\|v_{1}(x)-v_{2}(x)\right\| .
\end{aligned}
$$

Moreover there is a constant $k(\varepsilon)$ such that 


$$
k(\varepsilon) \cdot d(A(x, 1), A(x, 2)) \geqq\left\|v_{1}(x)-v_{2}(x)\right\|,
$$

so

$$
k(\varepsilon)\left\|h_{1}(x)-h_{2}(x)\right\| \geqq\left\|v_{1}(x)-v_{2}(x)\right\|=\left\|h_{1}(x) \circ f(x)-h_{2}(x) \circ f(x)\right\| .
$$

Therefore

$$
k(\varepsilon)\left\|h_{1}-h_{2}\right\|=\sup k(\varepsilon)\left\|h_{1}(x)-h_{2}(x)\right\| \geqq\left\|h_{1}(x) \circ f(x)-h_{2}(x) \circ f(x)\right\| .
$$

Lemma 9. Let $f$ be in $C\left(X, S^{2}\right)$ and $\phi$ be a frame function defined on $C\left(X, S^{2}\right)$. Let $M$ be a neighborhood of $f$ such that

$$
0 \leqq \sup \phi(M)-\inf \phi(M) \leqq \alpha .
$$

Then for each $g$ in $C\left(X, S^{2}\right)$ there is a neighborhood $U_{g}$ of $g$ such that

$$
0 \leqq \sup \phi\left(U_{g}\right)-\inf \phi\left(U_{g}\right) \leqq 4 \alpha
$$

Proof. (a) First we show that for each $h$ in $E(f)$ there is a neighborhood $V_{h}$ of $h$ such that

$$
0 \leqq \sup \phi\left(V_{h}\right)-\inf \phi\left(V_{h}\right)=2 \alpha .
$$

As in Lemma 6 one can assume that $f \equiv(0,0,1)$ and $h \equiv(0,1,0)$. For $\delta \in \boldsymbol{R}$ such that $0<\delta<1 / 10$ and $K_{\delta}(f) \subseteq M$, where $K_{\dot{\delta}}(f)$ is the open ball with radius $\delta$ and center $f$, define $u \in C\left(X, S^{2}\right)$ by $u \equiv$ $\left(0,\left[1+(3 / 2) \delta+(2 / 9) \delta^{2}\right]^{-1 / 2}[1+(1 / 3) \delta], \quad-(1 / 3) \delta\right)$. Define $A=K_{\delta / 9}(h) ;$ then $A \subseteq M(u, \delta / 9)$. So by Lemma 8 we have $\left\|h_{1} \circ u-h_{2} \circ u\right\| \leqq$ $k(\delta / 9)\left\|h_{1}-h_{2}\right\|$ for all $h_{1}$ and $h_{2}$ in $A$. Since $h_{1} \circ u$ and $h_{2} \circ u$ are elements of $M\left(h_{1}, \delta / 9\right)$ and $h_{1}$ and $h_{2}$ are elements of $M\left(h_{2} \circ u, \delta / 9\right)$, we have

$$
\begin{aligned}
& \left\|h_{1} \circ\left(h_{1} \circ u\right)-h_{2} \circ\left(h_{2} \circ u\right)\right\| \\
& \quad \leqq\left\|h_{1} \circ\left(h_{1} \circ u\right)-h_{1} \circ\left(h_{2} \circ u\right)\right\|+\left\|h_{1} \circ\left(h_{2} \circ u\right)-h_{2} \circ\left(h_{2} \circ u\right)\right\| \\
& \quad \leqq k(\delta / 9)\left\|h_{1} \circ u-h_{2} \circ u\right\|+k(\delta / 9)\left\|h_{1}-h_{2}\right\| \\
& \quad \leqq 2(1+k(\delta / 9))^{2}\left\|h_{1}-h_{2}\right\| .
\end{aligned}
$$

This implies that the map $F: A \rightarrow C\left(X, S^{2}\right)$ defined by $F(l)=l \circ(l \circ u)$ is continuous. Since $F(h)=f$ there is a neighborhood $V_{h}$ of $h$ such that $V_{h} \leqq A$ and $F\left(V_{h}\right) \subseteq K_{\delta / 9}(f)$. Choose $l \in V_{h}$. Then $l$ and $u$ are in $E(l \circ u)$, so

$$
\|F(l)-u \circ(l \circ u)\|=\|l \circ(l \circ u)-u \circ(l \circ u)\|=\|l-u\| .
$$

Thus

$$
\|f-u \circ(l \circ u)\| \leqq \delta / 3+\|l-u\| \leqq \delta / 3+\delta / 9+\|h-u\|<\delta,
$$


so $u \circ(l \circ u)$ is in $M$.

In addition, $\{l, l \circ u, l \circ(l \circ u)\}$ and $\{u, l \circ u, u \circ(l \circ u)\}$ are orthonormal bases, so we have

$$
\dot{\phi}(l)+\dot{\phi}(l \circ(l \circ u))=\dot{\phi}(u)+\dot{\phi}(u \circ(l \circ u)) .
$$

Let $l^{\prime}$ be another element of $V_{h}$. Then

$$
\dot{\phi}\left(l^{\prime}\right)+\dot{\phi}\left(l^{\prime} \circ\left(l^{\prime} \circ u\right)\right)=\dot{\phi}(u)+\dot{\phi}\left(u \circ\left(l^{\prime} \circ u\right)\right),
$$

and finally, by subtraction,

$$
\begin{aligned}
\mid \dot{\phi}(l)- & \phi\left(l^{\prime}\right)|\leqq| \dot{\phi}(u \circ(l \circ u))-\dot{\phi}\left(u \circ\left(l^{\prime} \circ u\right)\right) \mid \\
& +\left|\dot{\phi}(l \circ(l \circ u))-\dot{\phi}\left(l^{\prime} \circ\left(l^{\prime} \circ u\right)\right)\right| \leqq 2 \alpha .
\end{aligned}
$$

This completes the proof of (a).

(b) Now let $l$ be in $C\left(X, S^{2}\right)$. As in Lemma 6, we can assume that $f \equiv(0,0,1)$ and that $l$ is of the form $l=\left(l_{1}, 0, l_{3}\right)$. Let $h \equiv$ $(0,1,0)$; then $h \in E(f)$ and $l \in E(h)$. The proof of the lemma is completed by applying (a) twice.

Lemma 10. Let $\phi$ be a frame function defined on $C\left(X, S^{2}\right)$. Then $\dot{\phi}$ is continuous.

Proof. (The proof follows exactly the proof of Lemma 7.18, p. 154 of [5].) Let $\varepsilon>0$. Since every positive constant function is a frame function we can assume that inf $\phi\left(C\left(X, S^{2}\right)\right)=0$. Let $\eta>0$, $f \in C\left(X, S^{2}\right)$ be given such that $\phi(f)<\eta / 2$. As in Lemma 6 we can assume that $f \equiv(0,0,1)$. Define $F: C\left(X, S^{2}\right) \rightarrow C\left(X, S^{2}\right)$ by $F\left(\left(g_{1}, g_{2}\right.\right.$, $\left.\left.g_{3}\right)\right)=\left(-g_{2}, g_{1}, g_{3}\right) . \quad F$ is a homeomorphism which preserves orthogonality, so $\dot{\phi} \circ F$ is again a frame function. Define $\psi=\dot{\phi}+\phi \circ F$. Then $\psi$ is a frame function on $C\left(X, S^{2}\right)$, and is constant on $E(f)$. Moreover $\dot{\psi}(f)<\eta$. By Lemma 7 there is a $g$ in $N(f)$ and a neighborhood $U_{g}$ of $g$ such that

$$
0 \leqq \sup \psi\left(U_{g}\right)-\inf \psi\left(U_{g}\right) \leqq 3 \eta \text {. }
$$

By Lemma 9 there is a neighborhood $U_{f}$ of $f$ such that

$$
0 \leqq \sup \psi\left(U_{f}\right)-\inf \psi\left(U_{f}\right) \leqq 12 \eta,
$$

so

$$
0 \leqq \sup \psi\left(U_{f}\right) \leqq 13 \eta
$$

Thus

$$
0 \leqq \sup \dot{\phi}\left(U_{f}\right) \leqq 13 \eta,
$$

from which it follows that 


$$
0 \leqq \sup \phi\left(U_{f}\right)-\inf \phi\left(U_{f}\right) \leqq 13 \eta .
$$

By Lemma 9 there exists for each $h$ in $C\left(X, S^{2}\right)$ a neighborhood $U_{h}$ of $h$ such that

$$
0 \leqq \sup \phi\left(U_{h}\right)-\inf \phi\left(U_{h}\right) \leqq 52 \eta .
$$

Hence $\phi$ is continuous.

II. In this section let $H$ be a finite-dimensional complex Hilbert space of $\operatorname{dimension} \operatorname{dim} H \geqq 3$ and let $S$ be its unit sphere. Define $\mathscr{P}$ to be the set of all continuous functions on $X$ with values in the projections on $H$ and $\mathscr{P}_{1}$ to be the set of those members of $\mathscr{P}$ which have their values in the set of all one-dimensional projections. This section is devoted to the relationship between $C(X, S)$ and $\mathscr{P}_{1}$. For $f$ in $C(X, S)$ define the map $P_{f}: X \rightarrow \mathscr{P}(L(H))$ by $P_{f}(x)=P_{f(x)}$ (the projection on the one-dimensional subspace of $H$ spanned by $f(x)$ ).

Lemma 11. The map $f \rightarrow P_{f}$ is a map from $C(X, S)$ onto $\mathscr{P}_{1}$.

Proof. (a) Let $f \in C(X, S)$ and $x, y \in X$. We have

$$
\begin{aligned}
& \left\|P_{f}(x)-P_{f}(y)\right\|=\sup \left\{\left\|P_{f(x)} a-P_{f(y)} a\right\| \mid a \in S\right\} \\
& \quad=\sup \{\|(a, f(x)) f(x)-(a, f(y)) f(y)\| \mid a \in S\}=2\|f(x)-f(y)\|,
\end{aligned}
$$

so $P_{f}$ is a continuous map, so $P_{f} \in \mathscr{P}_{1}$.

(b) Let $P \in \mathscr{P}_{1}$. For each $x$ in $X$ select an $a_{x}$ in $(P(x) H) \cap S$ and define $f_{x}$ to be the constant function which maps $X$ to $a_{x}$. Then $f_{x}$ is in $C(X, S)$ and $\left\|P(x) f_{x}(x)\right\|=1$, so there is an open and closed neighborhood $U_{x}$ of $x$ such that $\left\|P(y) f_{x}(y)\right\|>1 / 2$ for all $y$ in $U_{x}$. Define $g_{x}: U_{x} \rightarrow S$ by $g_{x}(y)=\left\|P(y) f_{x}(y)\right\|^{-1} P(y) f_{x}(y)$. Then $g_{x}$ is continuous and for all $y \in U_{x}$ we have $P(y)=P_{g_{x}(y)}$. Since $\left(U_{x}\right)_{x \in X}$ is an open covering of $X$, there is a finite subcovering $\left(U_{x_{1}}, \cdots, U_{x_{n}}\right)$. Define $g \in C(X, S)$ by

$$
g(x)= \begin{cases}g_{x_{1}}(x), & \text { if } x \in U_{x_{1}} \\ g_{x_{k}}(x), & \text { if } x \in U_{x_{k}} \mid \cup\left\{U_{x_{i}} \mid i<k\right\} .\end{cases}
$$

Then we have $P=P_{g}$.

LEMMA 12. Let $n$ be a natural number and let $Q$ be in $\mathscr{P}$ such that $\operatorname{dim} Q(x)>n$ for all $x$ in $X$. Let $f_{1}, \cdots, f_{n}$ in $C(X, S)$ be pairwise orthogonal and such that $P_{f_{i}} \leqq Q$. Then there is a $g$ in $C(X, S)$ such that $g \perp f_{i}(1 \leqq i \leqq n)$ and $P_{g} \leqq Q$.

Proof. For each $x$ in $X$ select $a_{x}$ in $(Q(x) H) \cap S$ such that 
$a_{x} \perp f_{i}(x) \quad(1 \leqq i \leqq n)$. Define $g_{x}^{\prime}$ to be the constant mapping from $X$ to $a_{x}$. Then $g_{x}^{\prime}$ is in $C(X, S)$ and satisfies

$$
\left\|Q(x) g_{x}^{\prime}(x)\right\|=1
$$

and

$$
\sum_{i=1}^{n}\left(f_{i}(x), g_{x}^{\prime}(x)\right)^{2}=0 .
$$

Hence there exists an open and closed neighborhood $U_{x}$ of $x$ such that for $y \in U_{x}$ we have

$$
\left\|Q(y) g_{x}^{\prime}(y)\right\|>1 / 2
$$

and

$$
\sum_{i=1}^{n}\left(f_{i}(y), Q(y) g_{x}^{\prime}(y)\right)^{2}<1 / 4 .
$$

Define $h_{x}: U_{x} \rightarrow H$ by

$$
h_{x}(y)=Q(y) g_{x}^{\prime}(y)-\sum_{i=1}^{n}\left(Q(y) g_{x}^{\prime}(y), f_{i}(y)\right) f_{i}(y)
$$

and $g_{x}: U_{x} \rightarrow H$ by

$$
g_{x}(y)=\left\|h_{x}(y)\right\|^{-1} h_{x}(y) .
$$

As in the proof of Lemma 11 one can define a function $g$ with the desired properties.

Lemma 13. For $f_{1}$ and $f_{2}$ in $C(X, S)$ there exists a $\rho$ in $C(X, C)$ such that $\left(f_{1}, \rho f_{2}\right)$ is in $C(X, \boldsymbol{R})$.

Proof. Define $A=\left\{x \in X \mid\left(f_{1}(x), f_{2}(x)\right) \neq 0\right\}$. Then cl $A$ is open and closed. Define $\rho^{\prime}: A \rightarrow C$ by

$$
\rho^{\prime}(x)=\left|\left(f_{1}(x), f_{2}(x)\right)\right|^{-1}\left(f_{1}(x), f_{2}(x)\right) .
$$

Then $\rho^{\prime}$ is continuous and bounded on $A$; since $X$ is a hyperstonean space there is a (unique) continuous extension $\rho^{\prime \prime}$ of $\rho^{\prime}$ to $\mathrm{cl} A$. Finally define $\rho \in C(X, C)$ by $\rho=\rho^{\prime \prime}$ on $A$ and $\rho \equiv 1$ on the complement of $\mathrm{cl} A$.

Lemma 14. Let $P_{1}, P_{2}$ be in $\mathscr{P}_{1}$. Then there are functions $f_{1}, f_{2}, f_{3}$ in $C(X, S)$ such that the following hold:

(i) $f_{1}, f_{2}, f_{3}$ are pairwise orthogonal;

(ii) $P_{1}=P_{f_{1}}$;

(iii) there is an $f$ in $C(X, S)$ which is contained in the real submodule of $C(X, H)$ spanned by $f_{1}$ and $f_{2}$ and such that $P_{2}=P_{f}$. 
Proof. By Lemma 11 there are functions $g_{1}$ and $g_{2}$ in $C(X, S)$ such that $P_{1}=P_{g_{1}}$ and $P_{2}=P_{g_{2}}$. Define $f_{1}=g_{1}$. By Lemma 13 there is a $\rho$ in $C(X, \boldsymbol{C})$ such that $\left(f_{1}, \rho g_{2}\right)$ is in $C(X, \boldsymbol{R})$; define $f=\rho g_{2}$. Then $P_{2}=P_{f}$. Let $A=\left\{x \in X \mid\left(f_{1}, f\right) \neq 1\right\}$ and define $f_{2}^{\prime}: A \rightarrow S$ by

$$
f_{2}^{\prime}(x)=\left\|\left(f-\left(f, f_{1}\right) f_{1}\right)(x)\right\|^{-1}\left(f-\left(f, f_{1}\right) f_{1}\right)(x) .
$$

Then $f_{2}^{\prime}$ is continuous and bounded. $H$ is finite-dimensional, so the fact that $X$ is hyperstonean implies the existence of a continuous extension $f_{2}^{\prime \prime}$ of $f_{2}^{\prime}$ to the open and closed subset cl $A$ of $X$. By Lemma 12 there exists an $h$ in $C(\lceil\mathrm{cl} A, S)$ such that $h$ is orthogonal to the restriction of $f_{1}$ to the complement of cl $A$. Now define $f_{2}$ to be $f_{2}^{\prime \prime}$ on $\mathrm{cl} A$ and $h$ on the complement of cl $A$. Then $f_{1} \perp f_{2}$ and $f=\left(f, f_{1}\right) f_{1}+\left(f, f_{2}\right) f_{2}$. Moreover, $\left(f, f_{2}\right)$ is in $C(X, \boldsymbol{R})$, so $f$ is contained in the real module spanned by $f_{1}$ and $f_{2}$. Again by Lemma 12 there is an $f_{3}$ having the desired properties.

III. In this section let $\mathfrak{A}$ be a von Neumann algebra of type $\mathrm{I}_{n}$ where $n \in N$ and $n \geqq 3$. Then by [1], pp. 239-240, $\mathfrak{A}$ is isomorphic to $\mathfrak{B} \otimes L(H)$, where $\mathfrak{B}$ is an abelian von Neumann algebra, $H$ is an $n$-dimensional complex Hilbert space and $\otimes$ denotes the tensor product of von Neumann algebras. In particular, $\mathfrak{B}$ is isomorphic to a space $C(X, C)$, where $X$ is hyperstonean. So by [1], p. 24, Proposition 4, (ii) (and the fact that $\operatorname{dim} H=n<\infty$ ), $\mathfrak{A}$ is isomorphic to $C(X, L(H))$. We identify $C(X, L(H))$ with $\mathfrak{A}$ and $C(X, C)$ with the center $\mathfrak{B}$ of $\mathfrak{A}$.

If $m$ is a finitely additive $G$-measure on $\mathfrak{A}$ then the restriction of $m$ to $\mathfrak{B}$ induces a finitely additive measure on the open and closed subsets of $X$ and also induces a unique continuous positive linear form on $\mathfrak{B}$, denoted by $R(m)$.

Denote by $M$ the set of all finitely additive $G$-measures $m$ on $\mathfrak{A}$ with $m(\mathrm{Id})=1$, and let $M_{1}^{+}$denote the set of all positive linear forms on $\mathfrak{B}$ with norm 1 . Then $R$ is a map from $M$ onto $M_{1}^{+}$. Define $N=R^{-1}\left(\operatorname{ex} M_{1}^{+}\right.$) (ex $A$ the extreme points of $A$ ). For $m \in M$ denote by $S(m)$ the support (in $X$ ) of $R(m)$. If $m$ is in $N$ we also denote the single element of $S(m)$ by $S(m)$.

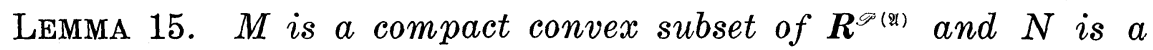
closed subset of $M$ containing ex $M$.

Proof. (a) $M$ is a closed convex subset of $[0,1]^{\mathscr{P ( x )}}$, so it is compact and convex.

(b) Equip $M_{1}^{+}$with the $w^{*}$-topology of $\mathfrak{B}^{*}=C(X, C)^{*}$. We show that $R$ is continuous, from which it follows that $N$ is a closed 
subset of $M$.

Let $\left(m_{i}\right)_{i \in I}$ be a net in $M$ converging to $m$, so $\left(m_{i}(P)\right)$ converges to $m(P)$ for all $P \in \mathscr{P}(\mathfrak{U})$. Now for $P \in \mathscr{P}(\mathfrak{B})$ we have $m(P)=$ $R(m)(P)$, so for all step functions $T$ in $\mathfrak{B}=C(X, C)$ we have $R\left(m_{i}\right)(T) \rightarrow R(m)(T)$. Let $f \in \mathfrak{B}$ and $\varepsilon>0$ be given. There is a step function $T \in \mathfrak{B}$ such that $\|f-T\| \leqq \varepsilon / 3$ and an $i_{0} \in I$ such that $\left|R\left(m_{i}\right)(T)-R(m)(T)\right|<\varepsilon / 3$ for all $i \geqq i_{0}$. So for all $i \geqq i_{0}$ we have

$$
\begin{aligned}
& \left|R(m)(f)-R\left(m_{i}\right)(f)\right| \leqq|R(m)(f)-R(m)(T)| \\
& \quad+\left|R(m)(T)-R\left(m_{i}\right)(T)\right|+\left|R\left(m_{i}\right)(T)-R\left(m_{i}\right)(f)\right|<\varepsilon .
\end{aligned}
$$

Thus $R\left(m_{i}\right)(f) \rightarrow R(m)(f)$, so $R$ is continuous.

(c) Let $m \in \operatorname{ex} M$, and assume that $R(m)$ is not in ex $M_{1}^{+}$. Then there exist $P_{1}, P_{2} \in \mathscr{P}(\mathfrak{B})$ such that $P_{1}+P_{2}=\mathrm{Id}$ and $\left.\lambda=m\left(P_{1}\right) \in\right] 0$, 1[. Define $m_{1}$ and $m_{2}$ by $m_{1}(Q)=\lambda^{-1} m\left(P_{1} Q\right)$ and $m_{2}(Q)=(1-\lambda)^{-1} m\left(P_{2} Q\right)$. Then $m=\lambda m_{1}+(1-\lambda) m_{2}$ is a nontrivial convex decomposition of $m$, which contradicts the assumption. We conclude that ex $M \subseteq N$.

LEMMA 16. Let $m$ be in $M$, let $U$ be an open and closed subset of $X$ containing $S(m)$, and let $Q=1_{U}$ (the indicator function of $U$ ); so $Q \in \mathfrak{B}$. Let $P_{1}, P_{2}$ be in $\mathscr{P}(\mathfrak{A})$ such that $P_{1} Q=P_{2} Q$, Then $m\left(P_{1}\right)=m\left(P_{2}\right)$.

Proof. $S(m)$ is the support of $R(m)$, so $m(Q)=1$ and $m(1-Q)=$ 0. Thus for all $P \in \mathscr{P}(\mathfrak{A})$ we have

$$
m(P)=m(P Q)+m(P(1-Q))=m(P Q) .
$$

LEMma 17. Let $m$ be in $N$ and let $P_{1}, P_{2} \in \mathscr{P}_{1}$ be such that $P_{1}(S(m))=P_{2}(S(m))$. Then $m\left(P_{1}\right)=m\left(P_{2}\right)$.

Proof. Choose $f_{1}, f_{2}, f_{3}$ and $f$ as in Lemma 14 . Let $g_{1}, g_{2}, g_{3}$ be the canonical module basis of $C\left(X, R^{3}\right)$. Define $I: C\left(X, R^{3}\right) \rightarrow C(X, H)$ by $I(h)=\left(h, g_{1}\right) f_{1}+\left(h, g_{2}\right) f_{2}+\left(h, g_{3}\right) f_{3}$. Then $I$ is an isometry of $C\left(X, \boldsymbol{R}^{3}\right)$ onto the real module spanned by $\left\{f_{1}, f_{2}, f_{3}\right\}$, and $I$ maps $C\left(X, S^{2}\right)$ into $C(X, S)$ (where $S$ is the unit sphere of $H$ ). Define a frame function $\phi$ on $C\left(X, S^{2}\right)$ by ${ }^{\prime} \phi(f)=m\left(P_{I(f)}\right)$. Now $P_{1}(S(m))=$ $P_{2}(S(m))$, so either $f_{1}(S(m))=f(S(m))$ or $f_{1}(S(m))=-f(S(m))$; we can assume that $f_{1}(S(m))=f(S(m)$ ) (otherwise take $-f$ instead of $f)$. Define $h=I^{-1}(f)$. Then we have $g_{1}(S(m))=h(S(m))$. For each $n \in N$ there exists an open and closed neighborhood $U_{n}$ of $S(m)$ such that

$$
\sup \left\{\left\|g_{1}(x)-h(x)\right\| \mid x \in U_{n}\right\}<1 / n .
$$

Define $h_{n} \in C\left(X, S^{2}\right)$ to be $h$ on $U_{n}$ and $g_{1}$ on the complement. Then 
$\left\|h_{n}-g_{1}\right\|<1 / n$, so $h_{n}$ converges to $g_{1}$. For $x \in U_{n}$ we have $h(x)=$ $h_{n}(x)$ and so also $f(x)=I(h)(x)=I\left(h_{n}\right)(x)$. By Lemma 16, $m\left(P_{2}\right)=$ $m\left(P_{I\left(h_{n}\right)}\right)=\phi\left(h_{n}\right)$ for all $n \in N$. Moreover, $m\left(P_{1}\right)=m\left(P_{f_{1}}\right)=\phi\left(g_{1}\right)$. So by Lemma 10 we conclude that $m\left(P_{1}\right)=\dot{\phi}\left(g_{1}\right)=\lim \phi\left(h_{n}\right)=\dot{\phi}(h)=$ $m\left(P_{2}\right)$.

For $P \in \mathscr{P}(\mathfrak{U})$ and $x \in X$ define $P_{x} \in \mathscr{P}(\mathfrak{A})$ by $P_{x}(y)=P(x)$ for all $y$ in $X$.

LEMMA 18. Let $m$ be in $N$ and $x=S(m)$. Then $m(P)=m\left(P_{x}\right)$ for all $P \in \mathscr{P}(\mathfrak{U})$.

Proof. The map $d: X \rightarrow N$, defined by $d(x)=\operatorname{trace} P(x)=\operatorname{dim} P(x)$ is continuous, so there is an open and closed neighborhood $U$ of $x$ such that $d$ is constant on $U$. By Lemma 16 we may assume $U=$ $X$. If $d \equiv 0$, then $P=0$ and $0=m(P)=m\left(P_{x}\right)$. Assume that $d \equiv n \in N$. By Lemma 12 there are $P_{1}, \cdots, P_{n} \in \mathscr{P}_{1}$ which are pairwise orthogonal and such that $P=\sum_{i=1}^{n} P_{i}$. Thus $m(P)=\sum_{i=1}^{n} m\left(P_{i}\right)$, and we may apply Lemma 17 to complete the proof.

LEMma 19. Let $m$ be in $N$. Then there is a unique state $f_{m}$ on $\mathfrak{A}$ extending $m$. The map $m \rightarrow f_{m}$ is continuous from $N$ to the set $S$ of all states of $\mathfrak{A}$ when $S$ is equipped with the $w^{*}$-topology of the dual of $\mathfrak{A}$.

Pr.oof. (a) Denote by $m^{\prime}$ the restriction of $m$ to $C \cdot 1_{X} \otimes L(H) \cong$ $L(H)$. Then $m^{\prime}$ is a $G$-measure on $L(H)$. Define $h_{m}$ to be the unique extension of $m^{\prime}$ to a state on $L(H)$. Define $I: \mathfrak{A} \rightarrow L(H)$ by $I(P)=$ $P(S(m))$. Then $I$ is a representation of $\mathfrak{A}$, and $f_{m}=h_{m} \circ I$ is a state on $\mathfrak{A}$. We show that $f_{m}$ extends $m$. Let $P \in \mathscr{P}(\mathfrak{A})$. Then we have $f_{m}(P)=h_{m} \circ I(P)=h_{m}(P(S(m)))=m^{\prime}(P(S(m)))=m\left(P_{S(m)}\right)=m(P)$, the last equality being justified by Lemma 18. Since the dimension of $H$ is finite, the linear combinations of elements must be dense in $\mathfrak{A}$ in the norm topology, so $f_{m}$ is unique.

(b) Let $\left(m_{i}\right)_{i \in I}$ be a net in $N$ converging to $m$. Then $\left(m_{i}^{\prime}\right)$ converges to $m^{\prime}$. As in the proof of Lemma 15 one can show that $\left(h_{m_{i}}\right)$ converges to $h_{m}$ and that $\left(f_{m_{i}}\right)$ converges to $f_{m}$.

Lemma 20. Let $m$ be in $M$. Then there is a unique state $f_{m}$ of $\mathfrak{A}$ extending $m$.

Proof. By Choquet's theorem there is a maximal measure $\mu$ on $M$ which represents $m$. By Lemma 15 the support of $\mu$ is in $N$. This and Lemma 19 suffice to settle the question of existence 
of an extension. The uniqueness follows as in Lemma 19.

IV. Proof of the theorem. By [1], p. 240, second paragraph and Proposition 2, $\mathfrak{A}$ is of the form $\mathfrak{A}=\Pi \mathfrak{A}_{n}$, where the $\mathfrak{A}_{n}$ are of type $I_{n}$ and $n$ is out of a fixed set of cardinals. By the assumption $n \neq 2$ for all $n$. Since $m$ is completely additive we can assume that $\mathfrak{A}$ is of type $\mathrm{I}_{n}$ and $n \neq 2$. If $n=1$, then $\mathfrak{A}$ is commutative, so we may assume that $n \geqq 3$.

We first prove the following claim:

If $P \in \mathscr{P}(\mathscr{U})$ is a finite projection, then the restriction (2) $m_{P}$ of $m$ to $P \mathscr{A} P$ has a unique extension to a state on $P$ Ar $P$.

Let $P \in \mathscr{P}(\mathfrak{A})$ be finite, that is, $P \mathscr{Q} P$ is a finite type I von Neumann algebra. As above we may restrict our attention to the case in which $P \mathfrak{A} P$ is of type $\mathrm{I}_{n}$, where now $n$ is a natural number. The case $n=1$ is the commutative case and for $n \geqq 3$ the claim follows by Lemma 20 . Only the case $n=2$ remains. Let $P \mathscr{A} P$ be of type $\mathrm{I}_{2}$. Then there are projections $P_{1}, P_{2}$ in $P \mathscr{A} P$ such that $P_{1}$ and $P_{2}$ are abelian relative to $P \mathscr{A} P, P_{1}$ is orthogonal to $P_{2}, P_{1}$ is equivalent to $P_{2}$ relative to $P \mathscr{A} P$, and $P_{1}+P_{2}=\operatorname{Id}_{P \mathscr{U} P}=P$. Since $P_{i} P=P P_{i}=P_{i}$ we have that $P_{1}$ and $P_{2}$ are abelian relative to $\mathfrak{A}$ and since $P \mathfrak{A} P \subseteq \mathfrak{A}$ they are equivalent relative to $\mathfrak{A}$ as well. Denote by $Z(Q)$ the central support of $Q \in \mathscr{P}(\mathfrak{A})$. Because of the additivity of $m$ we may assume that $Z(P)=1$. Let $Q=1-P$. Then by [1], p. 218, Théorème 1 , there exists a projection $G$ in $\mathfrak{B}$ such that

(a) $Q G \prec P_{1} G$, and (b) $P_{1}(1-G) \prec Q(1-G)$.

By (a) and [1], p. 123, Définition 3 and the succeeding paragraph there must be a projection $H$ in $\mathfrak{B}$ such that $H \leqq G$ and $Q G \sim P_{1} H$. Then $Z(Q G)=Z\left(P_{1} H\right)=Z\left(P_{1}\right) H=H$, so $Q(G-H)=0$ or $P(G-H)=$ $G-H$. Now $P \mathfrak{A} P$ is of type $\mathrm{I}_{2}$ and $\mathfrak{A}$ is of type $\mathrm{I}_{n}$ where $n>2$, so $G-H$ has to be 0 . So we have $Q G \sim P_{1} G$.

By (b) there is a $Q_{1} \leqq Q(1-G)$ such that $P_{1}(1-G) \sim Q_{1}$. Let $P_{3}=Q G+Q_{1}$. Then $P_{3} \sim P_{1}$, so $P_{3}$ is abelian and $P_{1}, P_{2}$ and $P_{3}$ are pairwise orthogonal. Define $P^{\prime}=P_{1}+P_{2}+P_{3}$. Thus $P^{\prime} \mathfrak{A} P^{\prime}$ is of type $\mathrm{I}_{3}$ and contains $P \mathscr{A} P$, so again we may apply Lemma 20. This completes the proof of (2).

Now define $F^{\prime}=\cup\{P \mathscr{A} P \mid P \in \mathscr{P}(\mathfrak{A}), P$ finite $\}$, and let $F$ be the vector subspace of $\mathfrak{A}$ which is spanned by $F^{\prime}$ and 1 ; that is, $F=$ $F^{\prime} \cup\left\{\lambda(1-A) \mid \lambda \in C\right.$ and $\left.A \in F^{\prime}\right\}$. Let $A$ be in $F$ and $P_{1}, P_{2}$ be finite projections in $\mathscr{P}(\mathfrak{A})$; denote by $Q$ their supremum in $\mathscr{P}(\mathfrak{U})$, which is again a finite projection. Then we have: $f_{Q}(A)$.

(i) If $A=P_{1} A P_{1}=P_{2} A P_{2}$, then $A=Q A Q$ and $f_{P_{1}}(A)=f_{P_{2}}(A)=$ 
(ii) If $A=P_{1} A P_{1}=\lambda(1-B)$ where $B=P_{2} B P_{2}$, then either $A=0$ or $1 \in Q \mathfrak{A} Q$, so $f_{P_{1}}(A)=f_{Q}(A)=\lambda\left(1-f_{P_{2}}(B)\right)$.

(iii) If $A=\lambda_{1}\left(1-P_{1} B_{1} P_{1}\right)=\lambda_{2}\left(1-P_{2} B_{2} P_{2}\right)$ then $\lambda_{1}\left(1-f_{P_{1}}\left(P_{1} B_{1} P_{1}\right)\right)=$ $\lambda_{2}\left(1=f_{P_{2}}\left(P_{2} B_{2} P_{2}\right)\right)$. These properties of the family $\left(f_{P}\right)$ allow us to define a linear form $f^{\prime}$ on $F$ by

$$
f^{\prime}(A)= \begin{cases}f_{P}(A), & \text { if } A \in P \mathfrak{U} P ; \\ \lambda\left(1-f_{P}(B)\right), & \text { if } A=\lambda(1-B) \text { and } B \in P \mathscr{A} P .\end{cases}
$$

We may assume that $m(1)=1$. Then we have the following:

(i) $F$ is self-adjoint and $1 \in F$;

(ii) $f^{\prime}(1)=1$;

(iii) $f^{\prime}(A) \geqq 0$ for all $A \in F \cap \mathfrak{Q Y}^{+}$;

(iv) $\left.f^{\prime}\left(A^{*}\right)=\overline{f^{\prime}(A}\right)$ for all $A \in F$.

We only show that (iii) is true for $A=\lambda(1-B)$ and $\lambda<0$. In this case $B \geqq 1$, so $\operatorname{supp} B \geqq 1$. Now $B=P B P$ for some finite $P$; that is, $1=P 1 P$. So $F=F^{\prime}=\mathfrak{A}$ and $f^{\prime}(A) \geqq 0$. By [2], p. 50, Lemme 2.10.1, the conditions (i)-(iv) ensure that there is a state $f$ on $\mathfrak{A}$ extending $f^{\prime}$. It remains to prove that $f$ is an extension of $m$; the normality of $f$ is then an immediate consequence of the complete additivity of $m$.

Let $P \in \mathscr{P}(\mathfrak{A})$. Then there exists a family $\left(P_{i}\right)_{i \in I}$ of pairwise orthogonal and finite projections in $\mathcal{A}$ and a subset $J \subseteq I$ such that

$$
\sum_{i \in I} P_{i}=1 \text { and } \sum_{i \in J} P_{i}=P \text {. }
$$

Then we have

$$
\begin{gathered}
f(P) \geqq \sum_{J} f\left(P_{i}\right)=\sum_{J} m\left(P_{i}\right)=m(P) . \\
f(1-P) \geqq \sum_{I \backslash J} f\left(P_{i}\right)=m(1-P),
\end{gathered}
$$

so

$$
f(P)=1-f(1-P) \leqq 1-m(1-P)=m(P) .
$$

By (3) and (4) we have $f(P)=m(P)$, so $f$ is an extension of $m$ and the proof is completed.

Acknowledgment. I am greatly indebted to Professor Kölzow for introducing me to this subject and for suggesting this particular area of investigation as well as for his constant encouragement.

\section{REFERENCES}

1. J. Dixmier, Les algébres d'operateurs dans l'espace Hilbertien, Gauthier-Villars, Paris, 1969.

2. _ Les $C^{*}$-algèbres et Leurs Representations, Gauthier-Villars, Paris, 1969. 
3. A. M. Gleason, Measures on the closed subspaces of a Hilbert space, J. Math. Mech., 6 (1957), 885-893.

4. A. A. Lodkin, Every measure on the projectors of a $W^{*}$-algebra can be extended to a state, Functional Anal. Appl., 8 (1974), 318-321.

5. V. S. Varadarajan, Geometry of Quantum Theory I, Van Nostrand, Princeton, N. J., 1968.

Received June 12, 1980 and in revised form October 8, 1980.

UNIVERSTTAT ERLANGEN-NURNBERG

D8520 Erlangen, W. Germany 


\section{PACIFIC JOURNAL OF MATHEMATICS}

\section{EDITORS}

DONALD BABBITT (Managing Editor)

University of California

Los Angeles, CA 90024

Hugo RossI

University of Utah

Salt Lake City, UT 84112

C. C. Moore and Arthur Agus

University of California

Berkeley, CA 94720
J. DugundJI

Department of Mathematics

University of Southern California

Los Angeles, CA 90007

R. FinN and J. MiLgRAM

Stanford University

Stanford, CA 94305

\section{ASSOCIATE EDITORS}
R. ARENS
E. F. BECKENBACH
B. H. NeumanN
F. WOLF
K. YoSHIDA

\section{SUPPORTING INSTITUTIONS}

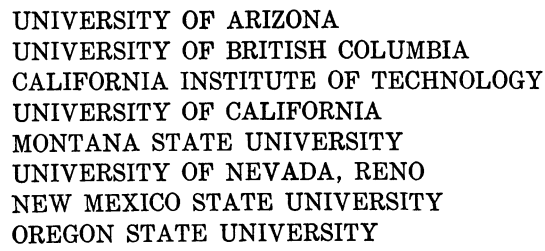

UNIVERSITY OF ARIZONA

UNIVERSITY OF BRITISH COLUMBIA CALIFORNIA INSTITUTE OF TECHNOLOGY UNIVERSITY OF CALIFORNIA MONTANA STATE UNIVERSITY

UNIVERSITY OF NEVADA, RENO NEW MEXICO STATE UNIVERSITY OREGON STATE UNIVERSITY

\author{
UNIVERSITY OF OREGON \\ UNIVERSITY OF SOUTHERN CALIFORNIA \\ STANFORD UNIVERSITY \\ UNIVERSITY OF AAWAII \\ UNIVERSITY OF TOKYO \\ UNIVERSITY OF UTAH \\ WASHINGTON STATE UNIVERSITY \\ UNIVERSITY OF WASHINGTON
}

The Supporting Institutions listed above contribute to the cost of publication of this Journal, but they are not owners or publishers and have no responsibility for its content or policies,

Mathematical parers intended for publication in the Pacific Journal of Mathematics should be in typed form or offset-reproduced, (not dittoed), double spaced with large margins. Please do not use built up fractions in the text of the manuscript. However, you may use them in the displayed equations. Underline Greek letters in red, German in green, and script in blue. The first paragraph or two must be capable of being used separately as a synopsis of the entire paper. Please propose a heading for the odd unmbered pages of less than 35 characters. Manuscripts, in triplicate, may be sent to any one of the editors. Please classify according to the scheme of Math. Reviews, Index to Vol. 39. Supply name and address of author to whom proofs should be sent. All other communications should be addressed to the managing editor, or Elaine Barth, University of California, Los Angeles, California, 90024 .

50 reprints to each author are provided free for each article, only if page charges have been substantially paid. Additional copies may be obtained at cost in multiples of 50 .

The Pacific Journal of Mathematics is issued monthly as of January 1966, Regular subscription rate: $\$ 114.00$ a year (6 Vol., 12 issues). Special rate: $\$ 57.00$ a year to individual members of supporting institution.

Subscriptions, orders for numbers issued in the last three calendar years, and changes of address shoud be sent to Pacific Journal of Mathematics, P.O. Box 969, Carmel Valley, CA 93924, U.S.A. Old back numbers obtainable from Kraus Periodicals Co., Route 100, Millwood, NY 10546.

\section{PUBLISHED BY PACIFIC JOURNAL OF MATHEMATICS, A NON-PROFIT CORPORATION}

Printed at Kokusai Bunken Insatsusha (International Academic Printing Co., Ltd.). 8-8, 3-chome, Takadanobaba, Shinjuku-ku, Tokyo 160, Japan.

Copyright (C) 1982 by Pacific Journal of Mathematics Manufactured and first issued in Japan 


\section{Pacific Journal of Mathematics}

Vol. 100, No. $2 \quad$ October, 1982

Kenneth F. Andersen, On the transformation of Fourier coefficients of

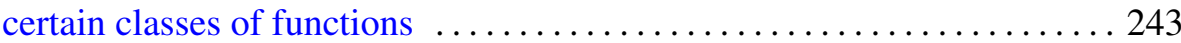

Steven Albert Bleiler, Realizing concordant polynomials with prime knots

Reinhard Bürger, Functions of translation type and solid Banach spaces of functions

Ulrich Daepp, The saturation of $k$-analytic rings and topological equivalence of associated analytic set germs .................. 271

Persi W. Diaconis and David Amiel Freedman, On the maximum difference between the empirical and expected histograms for sums . . . 287

David Amiel Freedman, On the maximum of scaled multinomial

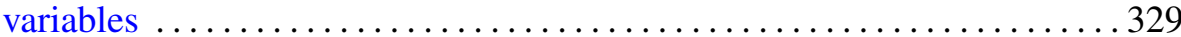

Persi W. Diaconis and David Amiel Freedman, On the difference between the empirical histogram and the normal curve, for sums. II ......... 359

Persi W. Diaconis and David Amiel Freedman, On the mode of an

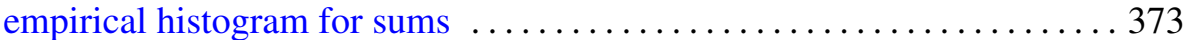

Jutta Hausen, Supplemented modules over Dedekind domains 387

Elyahu Katz, A moduli representation for the classification of twisted tensor

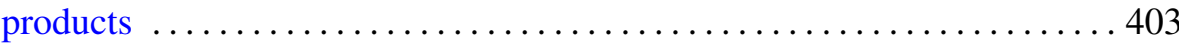

H. C. Madhekar and N. K. Thakare, Biorthogonal polynomials suggested by the Jacobi polynomials

Ted R. Pettis, Collections of covers of metric spaces 425

Ryōtarō Satō, Maximal functions for a semiflow in an infinite measure space

Michael Jay Stob, Invariance of properties under automorphisms of the lattice of recursively enumerable sets 